\title{
Review: corticosteroids do not reduce hospital length of stay or respiratory distress in infantile acute viral bronchiolitis
}

Patel H, Platt R, Lozano JM, et al. Glucocorticoids for acute viral bronchiolitis in infants and young children. Cochrane Database Syst $\operatorname{Rev} 2004 ;(3): C D 004878$.

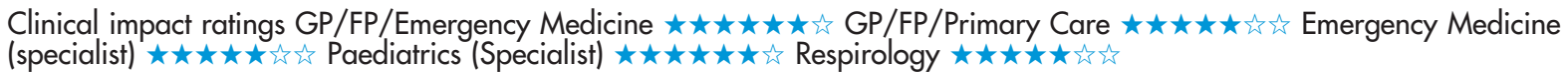

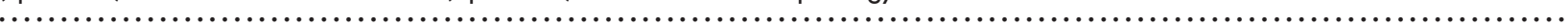

In infants and young children with acute viral bronchiolitis, do systemic corticosteroids shorten hospital length of stay (LOS) or improve respiratory distress more than placebo or no corticosteroid treatment?

\section{METHODS}

Data sources: Cochrane Central Register of Controlled Trials
(issue 3, 2003), Medline (1966 to September 2003), EMBASE/
Excerpta Medica (January 1990 to September 2003), Current
Contents (1998-2000), and SCISEARCH; reference lists of
general paediatric, infectious diseases, and respirology
textbooks; reference lists of retrieved studies and recent review
articles; meeting abstracts; authors of published abstracts; and
experts in the field.
$\begin{aligned} & \text { Study selection and assessment: randomised controlled trials } \\ & \text { (RCTs) in any language that evaluated systemic corticosteroids in } \\ & \text { infants and young children } \leqslant 24 \text { months of age with acute viral } \\ & \text { bronchiolitis. Study quality was assessed using the Jadad scale. } \\ & \text { Outcomes: hospital LOS and clinical severity scores (degree of } \\ & \text { respiratory distress over time). Hospital admissions were assessed } \\ & \text { in patients recruited from the emergency department (ED). }\end{aligned}$

\section{MAIN RESULTS}

13 RCTs met the selection criteria $(\mathrm{n}=1198$, age range $0-30 \mathrm{mo}) .10$ trials were set in inpatient paediatric hospital wards; 3 trials recruited patients from the ED and outcomes were measured in hospital or at home. Hospital LOS data were pooled in 7 RCTs of inpatients; groups did not differ for LOS (table). In 3 RCTs where recruitment and the start of intervention occurred in the ED, groups did not differ for Quebec, Canada. Hema.patel@muhc.mcgill.ca

Source of funding: not stated. hospital admission rates (table). Clinical score data pooled in 8 RCTs showed no difference between groups in day 3 clinical score (table).

\section{CONCLUSION}

In infants and young children with acute viral bronchiolitis, systemic corticosteroids do not shorten hospital length of stay or improve respiratory distress more than placebo or no corticosteroid treatment.

\section{Commentary}

$\mathrm{V}$ iral bronchiolitis is a common lower respiratory tract infection in infants and a frequent cause of hospital admission. The systematic review by Patel et al adds to a list of previous Cochrane reviews that all failed to show a significant effect on clinically relevant outcomes (eg, LOS or requirement for mechanical ventilation) for any of the treatments that are currently widely used (ie, inhaled bronchodilators, adrenaline or corticosteroids, immunoglobulins, and ribavirin). ${ }^{12}$

Given these data, only supportive treatment, if any, remains to be considered in infants with bronchiolitis. Such treatments include maintenance of adequate hydration and oxygenation. Thus, most infants are only admitted to hospital because no reliable tool exists to predict who will have apnoea or respiratory failure. Clinical experience suggests a trial of continuous positive airway pressure in infants with respiratory failure, but again insufficient evidence exists from RCTs for this treatment. ${ }^{3}$ There remains an urgent need for large, well designed RCTs in this field. Christian F Poets, MD University Hospital Tübingen, Germany Cochrane Databas, Syst Rev 2004.(1):CD003123.

2 Fitzgerald DA, Kilham HA. Bronchiolitis: assessment and evidence-based management. Med J Aust 2004; 180:399-404.

3 Shah PS, Ohlsson A, Shah JP. Continuous negative extrathoracic pressure or continuous positive airway pressure for acute hypoxemic respiratory failure in children. Cochrane Database Syst Rev 2003;(3):CD003699.

Systemic corticosteroids $v$ placebo or no corticosteroid treatment in infants or young children with acute viral bronchiolitis*

\begin{tabular}{lll}
\hline Outcomes & Number of trials $(\mathbf{n})$ & Weighted mean difference $(95 \% \mathrm{Cl})$ \\
\hline Hospital length of stay $(\mathrm{d}) \dagger$ & $7(472)$ & $-0.38(-0.81$ to 0.05$)$ \\
\hline & & Odds ratio (Cl) \\
\hline Hospital admission ratef & $3(156)$ & $1.05(0.23$ to 4.87$)$ \\
\hline & & Standardised mean difference (CI) \\
\hline Day 3 clinical score & $8(309)$ & $-0.20(-0.73$ to 0.32$)$ \\
\hline
\end{tabular}

${ }^{*} \mathrm{Cl}$ defined in glossary; a random effects model was used. All differences are not significant

tPatients were inpatients on paediatric hospital wards.

‡Patients were recruited from the emergency department. 